\title{
Effects of bisphosphonates on osteoclastogenesis in RAW264.7 cells
}

\author{
KEIGO ABE ${ }^{1,2}$, YOSHITAKA YOSHIMURA ${ }^{2}$, YOSHIAKI DEYAMA ${ }^{2}$, TAKASHI KIKUIRI ${ }^{3}$, \\ TOMOKAZU HASEGAWA ${ }^{5}$, KANCHU TEI ${ }^{4}$, HISASHI SHINODA ${ }^{6}$, \\ KUNIAKI SUZUKI $^{2}$ and YOSHIMASA KITAGAWA ${ }^{1}$
}

\author{
Departments of ${ }^{1}$ Oral Diagnosis and Oral Medicine, ${ }^{2}$ Molecular Cell Pharmacology, ${ }^{3}$ Pediatric Dentistry, ${ }^{4}$ Oral and \\ Maxillofacial Surgery, Hokkaido University Graduate School of Dental Medicine, Kita-ku, Sapporo 060-8586; \\ ${ }^{5}$ Department of Pediatric Dentistry, Faculty of Dentistry, Tokushima University, Tokushima 770-8504; \\ ${ }^{6}$ Department of Applied Pharmacology, Tohoku University Graduate School of Dentistry, Sendai 980-8575, Japan
}

Received December 28, 2011; Accepted February 17, 2012

DOI: $10.3892 / \mathrm{ijmm} .2012 .952$

\begin{abstract}
Bisphosphonates are used as therapeutic agents for the management of osteoporosis and other bone diseases. However, the precise effects and mechanisms of bisphosphonates on osteoclastogenesis are unclear, as previous studies have reported contradictory findings and no studies have circumstantially assessed the effects of bisphosphonates on osteoclastogenesis. Therefore, the aim of this study was to determine the effects of bisphosphonates on osteoclastogenesis in RAW264.7 (RAW) cells. To examine the direct effects of bisphosphonates on osteoclast differentiation via receptor activator of nuclear factor- $\mathrm{kB}$ (RANK) ligand (RANKL), RAW cells were cultured with bisphosphonates. Addition of bisphosphonates to RAW cells led to a significant decrease in the number of osteoclasts and large osteoclasts ( $\geq 8$ nuclei) in a bisphosphonate concentration-dependent and time-dependent manner. The cytotoxicity of non-nitrogen-containing bisphosphonates was specific to osteoclasts, while nitrogen-containing bisphosphonates were cytotoxic and induced cell death in both osteoclasts and RAW cells. Resorption activity was significantly diminished by treatment with bisphosphonates, thus confirming that bisphosphonates impair the absorptive activity of osteoclasts. We also investigated the effects of bisphosphonates on the mRNA expression of genes associated with osteoclastogenesis, osteoclast-specific markers and apoptosis-related genes using quantitative real-time PCR. The results suggest that bisphosphonates suppress osteoclast differentiation and infusion, and induce osteoclast apoptosis. With regard to osteoclast apoptosis induced by bisphosphonates, we
\end{abstract}

Correspondence to: Dr Yoshitaka Yoshimura, Department of Molecular Cell Pharmacology, Hokkaido University Graduate School of Dental Medicine, Kita 13, Nishi 7, Kita-ku, Sapporo 060-8586, Japan E-mail: yoshi@den.hokudai.ac.jp

Key words: non-nitrogen-containing bisphosphonates, nitrogencontaining bisphosphonates, osteoclast further investigated the detection of DNA fragmentation and Caspase-Glo 3/7 assay. DNA fragmentation was confirmed after treatment with bisphosphonates, while caspase-3/7 activity increased significantly when compared with controls. In conclusion, bisphosphonates directly inhibited RANKLstimulated osteoclast differentiation and fusion in RAW cells. It was confirmed that bisphosphonates impair osteoclast resorption activity and induce apoptosis. The effects of nonnitrogen-containing bisphosphonates were also specific to osteoclasts, while nitrogen-containing bisphosphonates were cytotoxic and induced cell death in both osteoclasts and RAW cells.

\section{Introduction}

Osteoclasts are members of the monocyte/macrophage lineage and are formed by fusion of their mononuclear cellular precursors. The receptor activator of nuclear factor- $\mathrm{kB}$ (RANK) ligand (RANKL) is the most essential cytokine for the process of osteoclast differentiation and activation (1). RANKL is a member of the tumor necrosis factor (TNF) family and is expressed as a membrane-bound protein in osteoblasts and stromal cells (2). The receptor for RANKL, termed RANK, is induced by macrophage colony-stimulating factor (M-CSF) in osteoclast precursor cells. M-CSF is also an essential factor for the survival of osteoclast precursor cells and for the formation of osteoclasts (3). The pre-osteoclastic RAW264.7 (RAW) cells we used in this study express endogenous RANK and are able to differentiate into osteoclasts following RANKL stimulation without M-CSF.

Bisphosphonates are a class of compounds that contain the $\mathrm{P}-\mathrm{C}-\mathrm{P}$ substructure instead of the P-O-P moiety present in pyrophosphate (4), and to this P-C-P backbone, two side chains are attached (Fig. 1). It has been reported that the pharmacological characteristics of bisphosphonates vary depending on the nature of the side chains (5). Bisphosphonates are also classified into nitrogen-containing and non-nitrogen-containing bisphosphonates. Non-nitrogen-containing bisphosphonates, which lack nitrogen and contain short $R_{1}$ and $R_{2}$ side chains, are incorporated into non-hydrolysable ATP analogues. These metabolites accumulate in the cell cytoplasm, consequently inhibiting numerous 


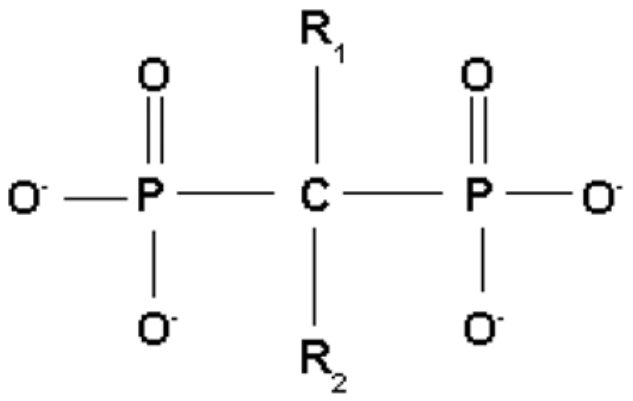

Figure 1. Structure of bisphosphonates. Bisphosphonates possess a P-C-P backbone, to which two side chains $\left(R_{1}\right.$ and $\left.R_{2}\right)$ are attached.

intracellular metabolic enzymes, resulting in detrimental effects on cell function and survival, while nitrogen-containing bisphosphonates block protein prenylation by inhibiting the mevalonate pathway (4). Via both of these mechanisms, osteoclast signaling is disrupted. In clinical practice, bisphosphonates have therefore been used as therapeutic agents in the management of osteoporosis and other bone diseases, such as hypercalcemia, metastatic bone disease and Paget's disease (6).

However, the precise effects and mechanisms of bisphosphonate action on osteoclastogenesis are unclear. For example, bisphosphonates directly inhibit osteoclast adhesion on the bone margin where resorption takes place. Bisphosphonates also act indirectly on osteoblasts, suppressing osteoclast differentiation (7). In contrast, Weinstein et al (8) reported that bisphosphonate-induced inhibition of bone resorption did not require osteoclast apoptosis. In addition, administration of bisphosphonates to beagles gave a higher rather than lower number of osteoclasts, apparently by prolonging osteoclast lifespan. These observations suggested that bisphosphonates inhibit bone resorption without decreasing the number of osteoclasts and may even increase the number of these cells in cancellous bone. Despite these apparently contradictory findings, there have been no studies circumstantially assessing the effects of bisphosphonates on osteoclastogenesis. Therefore, the aim of this study was to determine the effects of bisphosphonates on osteoclastogenesis in RAW cells.

\section{Materials and methods}

Reagents (bisphosphonates). The non-nitrogen-containing bisphosphonates used were etidronate (Dainippon Sumitomo Seiyaku, Japan) and TRK-530 (Shouwa Yakuhin, Japan). TRK-530 is a recently developed novel synthetic bisphosphonate with an anti-oxidant methylthio-phenylthio side chain (5). The nitrogen-containing bisphosphonates used were alendronate (Sigma-Aldrich, St. Louis, MO, USA), risedronate (Procter and Gamble Pharmaceutics, Wood Corners Laboratories, Norwich, NY, USA) and zoledronate (Novartis Pharma AG, Switzerland). Zoledronate is the most potent commercially available bisphosphonate when given systemically through subcutaneous injections (9). All bisphosphonates were stocked at a range of concentrations $(0$, $0.01,0.1,1.0$ and $10 \mu \mathrm{M})$.

Cell culture. The murine monocyte/macrophage cell line RAW (ATCC, Manassas, VA, USA) was used for osteoclast precursor cells. RAW cells differentiate into osteoclast-like cells in the presence of RANKL. Cells were grown in Dulbecco's modified Eagle's medium (DMEM; Wako Pure Chemical Industries, Ltd., Japan), supplemented with $10 \%$ (v/v) heat-inactivated fetal bovine serum (FBS) (Invitrogen, Frederick, MD, USA) and $66.7 \mu \mathrm{g} / \mathrm{ml}$ kanamycin-sulfate (Meiji Seika, Japan) at $37^{\circ} \mathrm{C}$ in a humidified atmosphere of $95 \%$ air and $5 \% \mathrm{CO}_{2}$. Cells were seeded onto 100-mm standard dishes (Falcon ${ }^{\mathrm{TM}}$; BectonDickinson Labware, Franklin Lakes, NJ, USA).

For osteoclast differentiation, RAW cells were transferred to 48 -well culture plates (Falcon) at a density of $0.5 \times 10^{4}$ cells/ well with $\alpha$-minimum essential medium ( $\alpha$-MEM; Wako Pure Chemical Industries, Ltd.) supplemented with $10 \%$ heatinactivated FBS, $50 \mathrm{ng} / \mathrm{ml}$ RANKL (Oriental Yeast, Japan), $284 \mu \mathrm{M}$ L-ascorbic acid 2-phosphate (Sigma-Aldrich), $2 \mathrm{mM}$ L-alanyl-L-glutamine (Sigma-Aldrich) and $66.7 \mu \mathrm{g} /$ $\mathrm{ml}$ kanamycin-sulfate at $37^{\circ} \mathrm{C}$ in a humidified atmosphere of $95 \%$ air and $5 \% \mathrm{CO}_{2}$ prior to treatment with different reagents. Medium containing these reagents was replaced every other day.

Tartrate-resistant acid phosphatase (TRAP) staining. Cells cultured for a given period were washed with PBS and fixed in $10 \%$ neutral formalin. They were then washed with distilled water and stained with fast red violet LB salt (SigmaAldrich). After washing, TRAP-positive cells with $\geq 2$ nuclei were considered to be osteoclast-like cells and osteoclasts with $\geq 8$ nuclei were considered to be large osteoclasts (10). Osteoclasts were counted under a light microscope.

Analysis of cytotoxicity. The cytotoxicity of bisphosphonates was analyzed using the Cell Counting kit-8 (Dojindo Molecular Technologies, Inc., Kumamoto, Japan). RAW cells were seeded on 96-well plates (Falcon) at a density of $0.2 \times 10^{4}$ cells/ well with or without bisphosphonates. After 6 days of culture, medium was replaced with new medium $(100 \mu \mathrm{l})$ and $10 \mu \mathrm{l}$ of Cell Counting kit- 8 solution was added. After incubation for $2 \mathrm{~h}$ at $37^{\circ} \mathrm{C}$, absorbance was measured at $450 \mathrm{~nm}$ using a microplate reader (Model 550, Bio-Rad, USA) and the background reading (medium) was subtracted.

Quantitative real-time PCR. RAW cells were seeded onto 6 -well plates (Falcon) at a density of $5 \times 10^{4}$ cells/well. After culture for 3 days (osteoclast differentiation) or 5 days (mature osteoclasts), RAW cells were treated with bisphosphonates for $24 \mathrm{~h}$. Total-RNA was extracted using TRIzol (Invitrogen), in accordance with the manufacturer's instructions. cDNA was synthesized from total-RNA using ReverTra Ace reverse transcriptase (Toyobo Co., Japan) and oligo(dt) primers.

For analysis of osteoclastogenic protein mRNAs, such as NFATc1, dendritic cell-specific transmembrane protein (DC-STAMP), the osteoclast-specific marker TRAP and the apoptosis-related genes Fas, Bcl-xL, caspase-3 (Casp3) and caspase-8 (Casp8), the following specific primer sets were used: NFATc1 (Mm00479445_m1); DC-STAMP (Mm01168058_ m1); TRAP (Mm00475698_m1); Fas (Mm00433237_m1); Casp3 (Mm01195085_m1); Casp8 (Mm00802247_m1); Bcl-xL (Mm00437783_m1); and glyceraldehyde 3-phosphate dehydrogenase (GAPDH) (Mm99999915_g1). Primers were designed using the Primer Express program (Applied Biosystems, Foster 




Figure 2. Number of TRAP-positive multinucleated osteoclasts varies depending on duration of culture. TRAP-positive multinucleated osteoclasts were counted after culturing with RANKL (50 ng/ml) for 8 days. Results are shown as means \pm SD for four independent experiments.

City, CA, USA) and were purchased from Applied Biosystems. Quantitative real-time PCR was performed using the ABI 7300 (Applied Biosystems).

We used the comparative $\mathrm{Ct}$ method to calculate mRNA expression. We verified the $\mathrm{Ct}$ values of both the calibrator and samples of interest by normalization against GAPDH. The comparative $\mathrm{Ct}$ method is also known as the $2^{-\Delta \Delta \mathrm{Ct}}$ method, in which, $\Delta \mathrm{Ct}=\Delta \mathrm{Ct}$ sample- $\Delta \mathrm{Ct}$ reference. $\Delta \mathrm{CT}$ sample is the $\mathrm{Ct}$ value for the samples normalized against the endogenous housekeeping gene, and $\Delta \mathrm{Ct}$ reference is the $\mathrm{Ct}$ value for the calibrator, which was also normalized against the endogenous housekeeping gene.

Pit assay. Pit formation assay was analyzed using the Corning Osteo Assay Surface Plate (Corning, Inc., Corning, NY, USA). RAW cells were seeded onto 24-well plates at a density of $1.0 \times 10^{4}$ cells/well with or without bisphosphonates. After 10 days of culture, plates were observed under a light microscope and pit area was compared.

Detection of DNA fragmentation in agarose gels. RAW cells were seeded onto standard 100-mm dishes. After 3 days of culture, RAW cells were treated with bisphosphonates for $24 \mathrm{~h}$. Cells were centrifuged and washed once with cold PBS(-). Cell pellets were lysed in $600 \mu \mathrm{l}$ of buffer consisting of $10 \mathrm{mM}$ Tris-HCI, $10 \mathrm{mM}$ EDTA and $0.2 \%$ Triton X-100 (pH 7.5). After $10 \mathrm{~min}$ on ice, lysates were centrifuged $(13,000 \mathrm{x} \mathrm{g})$ for $10 \mathrm{~min}$ at $4^{\circ} \mathrm{C}$. Subsequently, the supernatant (containing RNA and fragmented DNA, but not intact chromatin) was extracted first with phenol, followed by phenol-chloroform:isoamyl alcohol (24:1). The aqueous phase was made up to $300 \mathrm{mM}$ $\mathrm{NaCl}$ and nucleic acids were precipitated with 2 volumes of ethanol. The pellet was rinsed with $70 \%$ ethanol, air-dried and dissolved in $15 \mu \mathrm{l}$ of $10 \mathrm{mM}$ Tris-HCI-1 mM EDTA (pH 7.5). After digesting RNA with RNase A $(0.6 \mathrm{mg} / \mathrm{ml})$ at $37^{\circ} \mathrm{C}$ for $30 \mathrm{~min}$, samples were electrophoresed on a $2 \%$ agarose gel with Boyer's buffer (50 mM Tris-HCI, $20 \mathrm{mM}$ sodium acetate, $2 \mathrm{mM}$ EDTA and $18 \mathrm{mM} \mathrm{NaCl}, \mathrm{pH}$ 8.05). DNA was then visualized by ethidium bromide staining (11).
Caspase-3/7 activity. RAW cells were seeded on a 96-well plate (LumiNunc ${ }^{\mathrm{TM}}$ plates) (Nalge Nunc International) at a density of $0.33 \times 10^{4}$ cells/well. After 3 days, RAW cells were treated with bisphosphonates for $24 \mathrm{~h}$. Caspase-Glo 3/7 assay (Promega, Madison, WI, USA) was then performed in accordance with the manufacturer's instructions. Luminescence was measured using a luminometer (Wallac 1420 ARVOsx; Perkin Elmer Co., Ltd., USA). Blank values were subtracted, and fold increase in activity was calculated based on activity measured in untreated cells.

Statistical analysis. All data are expressed as means \pm SD. Comparisons between samples of interest and respective controls were analyzed by two-tailed unpaired Student's t-test. P-values of $<0.05$ were considered to be significant.

\section{Results}

Number of TRAP-positive multinucleated osteoclasts varies with duration of culture time. TRAP-positive multinucleated osteoclasts were counted after culturing with RANKL (50 ng/ $\mathrm{ml}$ ) for 8 days. Numerous osteoclasts were observed after 4 days of culture, and the number continued to increase until 6 days of culture (Fig. 2).

Effects of bisphosphonates on number of TRAP-positive multinucleated osteoclasts. We analyzed the effects of bisphosphonates on osteoclast differentiation by incubating RAW cells with different concentrations of bisphosphonates $(0,0.01,0.1$, 1.0 and $10 \mu \mathrm{M}$ ) together with RANKL, and then counting both the number of osteoclasts and the number of large osteoclasts after 6 days of culture. Bisphosphonates decreased the number of osteoclasts in a concentration-dependent manner. Similarly, the number of large osteoclasts with $\geq 8$ nuclei was decreased by bisphosphonates (Fig. 3).

We also analyzed the effects of bisphosphonates $(10 \mu \mathrm{M})$ by changing the administration duration (1, 3 and 5 days), and counting both the number of osteoclasts and the number of large osteoclasts after 6 days of culture. Bisphosphonates decreased the number of osteoclasts in a time-dependent manner. Similarly, the number of large osteoclasts with $\geq 8$ nuclei was decreased by bisphosphonates (Fig. 4).

Cytotoxicity of bisphosphonates for RAW cells and osteoclasts. First, we analyzed cytotoxicity of bisphosphonates $(10 \mu \mathrm{M})$ for RAW cells without RANKL. Non-nitrogen-containing bisphosphonates (etidronate and TRK-530) were not cytotoxic and did not induce cell death. However, nitrogen-containing bisphosphonates (alendronate, risedronate and zoledronate) were cytotoxic and induced cell death (Fig. 5A).

Next, we analyzed the cytotoxicity of bisphosphonates $(10 \mu \mathrm{M})$ with RANKL. Non-nitrogen-containing bisphosphonates were cytotoxic and induced cell death, while nitrogen-containing bisphosphonates were very cytotoxic and induced cell death. Greater cytotoxicity was observed with RANKL (Fig. 5B).

Pit assay. We investigated the resorption activity of osteoclasts treated with bisphosphonates (zoledronate or TRK-530; $10 \mu \mathrm{M})$, as compared with a control group, using the Corning 

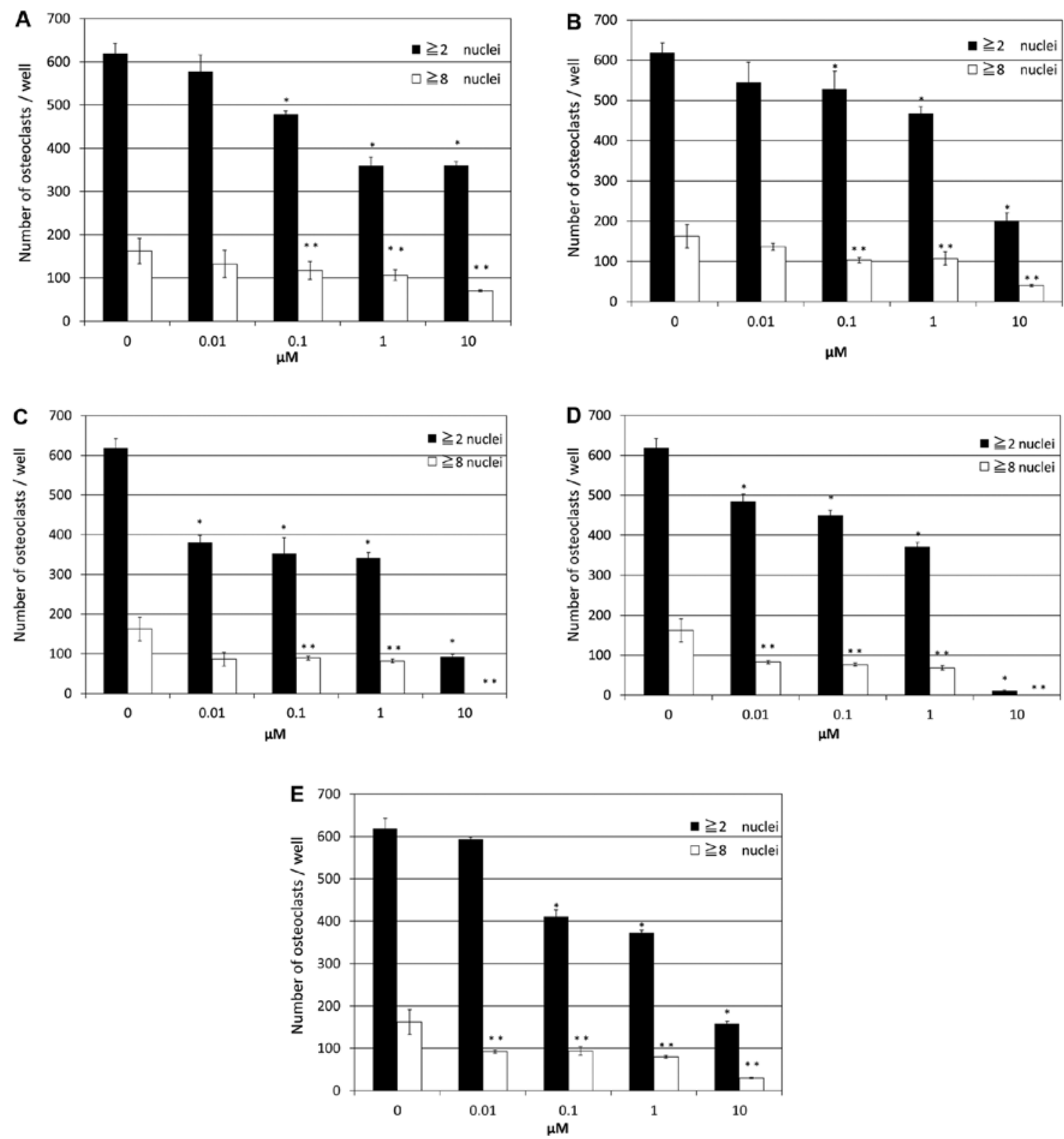

Figure 3. Effects of bisphosphonates $(0,0.01,0.1,1.0$ or $10 \mu \mathrm{M})$ on osteoclast differentiation. We incubated RAW264.7 cells with different concentrations of bisphosphonates $(0,0.01,0.1,1.0$ or $10 \mu \mathrm{M})$ together with RANKL, and counted both the number of osteoclasts and large osteoclasts after 6 days of culture. (A) etidronate, (B) alendronate, (C) risedronate, (D) zoledronate and (E) TRK-530. Results are shown as means \pm SD for four independent experiments. "P $<0.05$, significant difference from control osteoclasts $\left(\geq 2\right.$ nuclei); and ${ }^{\#} \mathrm{P}<0.05$, significant difference from control large osteoclasts ( $\geq 8$ nuclei).

Osteo Assay Surface Plate. Pit area was confirmed to be $56 \%$ in the controls, while zoledronate treatment gave a pit area of $0 \%$ and TRK-530 gave a pit area of $0.5 \%$ (Fig. 6).

Effects of bisphosphonates on mRNA expression of osteoclastic differentiation genes and osteoclast-specific markers in RAW cells. We investigated the effects of bisphosphonates on the mRNA expression of genes associated with osteoclastogenesis and osteoclast-specific markers. After 3 or 5 days of culture, RAW cells were treated with bisphosphonates (zoledronate or TRK-530) $(10 \mu \mathrm{M})$, or were left untreated (control group), for $0,1,3,6,12$ and $24 \mathrm{~h}$. We analyzed the mRNA expression of these genes using real-time PCR analysis. Genes examined for mRNA expression were NFATc1, DC-STAMP and TRAP. After 3 days of culture, bisphosphonates reduced the mRNA expression of TRAP. In addition, the mRNA expression of
DC-STAMP decreased. However, bisphosphonates did not affect the mRNA expression of NFATc1.

After 5 days of culture, bisphosphonates reduced the mRNA expression of NFATc1 as well as TRAP and DC-STAMP (Fig. 7).

Effects of bisphosphonates on mRNA expression of genes with reference to apoptosis in $R A W$ cells. We further analyzed the effects of bisphosphonates on the mRNA expression of genes with reference to apoptosis. After 3 or 5 days of culture, RAW cells were treated with bisphosphonates (zoledronate or TRK-530) $(10 \mu \mathrm{M})$, or were left untreated (control group) for $0,1,3,6,12$ and $24 \mathrm{~h}$. Genes examined for mRNA expression were Fas, Casp3, Casp8 and Bcl-xL. After 3 days of culture, bisphosphonates showed increased mRNA expression of Fas after treatment for 3 and $6 \mathrm{~h}$. In addition, the mRNA expression of Casp8 was higher with bisphosphonate treatment for 



Figure 4. Effects of bisphosphonates on osteoclast differentiation after treatment for various durations (1,3 or 5 days). We incubated RAW264.7 cells for various time durations (1, 3 or 5 days) with RANKL and bisphosphonates $(10 \mu \mathrm{M})$, and counted both osteoclasts and large osteoclasts after 6 days of culture. 1 day: after 5 days of culture, bisphosphonates were added for 24 h. 3 days: after 3 days of culture, bisphosphonates were added for 72 h. 5 days: bisphosphonates were added for $120 \mathrm{~h}$ from Day 1 . Control was treated with distilled water. (A) Number of TRAP-positive osteoclasts ( $\geq 2$ nuclei). (B) Number of TRAP-positive osteoclasts ( $\geq 8$ nuclei). Results are shown as means \pm SD for four independent experiments. ${ }^{*} \mathrm{P}<0.05$, significant difference vs. controls.

A

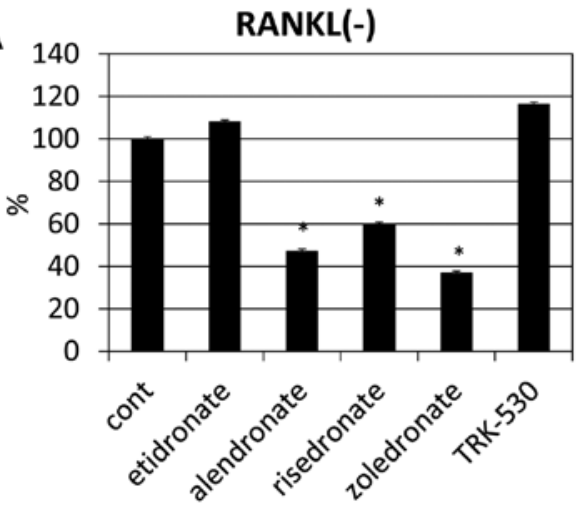

B

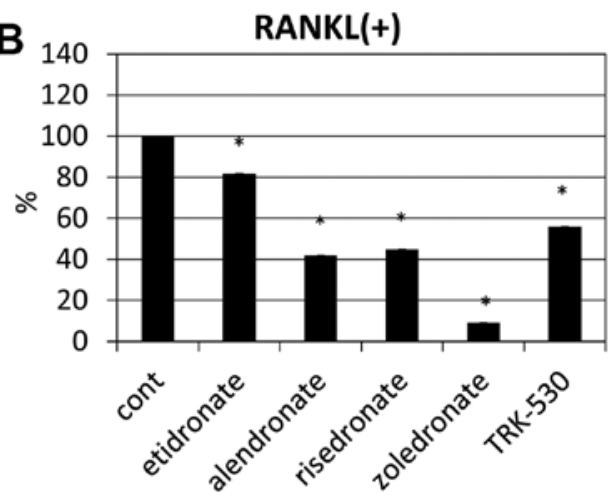

Figure 5. Effects of bisphosphonate cytotoxicity on osteoclast differentiation. The cytotoxicity of bisphosphonates was analyzed. After 6 days of culture, absorbance was measured at $450 \mathrm{~nm}$ using a microplate reader. Control was treated with distilled water. (A) RAW264.7 cells cultured without RANKL. (B) RAW264.7 cells cultured with RANKL. Results are shown as means \pm SD for four independent experiments. "P<0.05, significant difference vs. controls.

12 h. Meanwhile, the mRNA expression of Bcl-xL was lower with bisphosphonate treatment for $6 \mathrm{~h}$.

After 5 days of culture, bisphosphonates increased the mRNA expression of Fas after treatment for 1, 3 and $6 \mathrm{~h}$. In addition, the mRNA expression of Casp3 was higher with bisphosphonate treatment for $3 \mathrm{~h}$. The mRNA expression of Bcl-xL was lower with bisphosphonates treatment at all time points, except $1 \mathrm{~h}$ (Fig. 8).

Detection of the DNA fragmentation in agarose gels and caspase-3/7 activity. We further analyzed apoptosis of osteoclasts induced by bisphosphonates.

After 3 days of culture, RAW cells were treated with bisphosphonates (zoledronate or TRK-530; $10 \mu \mathrm{M}$ ), or were left untreated (control group), for 1 day.

DNA fragmentation was confirmed after treatment with both bisphosphonates, but was not observed in controls (Fig. 9). After treatment with bisphosphonates, caspase-3/7 activity increased significantly when compared with controls (Fig. 10).

\section{Discussion}

Bisphosphonates have potent osteoclastic inhibitory effects and are used to control progressive bone loss in Paget's disease and osteoporosis by increasing bone density and preventing possible pathological fractures (12). Bisphosphonates are also thought to play a major role in skeletal health preservation in cancer patients owing to their ability to inhibit bone resorption (13). Patients with cancer are at risk of bone loss induced by their cancer treatments, and are known to experience skeletal metastases and serious pathological fractures. In recent studies, some authors have proposed additional direct antitumor effects of bisphosphonate therapy, possibly through the stimulation of tumor cell apoptosis and inhibition of their invasion and adhesion, thereby delaying or preventing skeletal metastasis (14). However, in recent years, serious adverse effects have been viewed with suspicion, and bisphosphonate-related osteonecrosis of the jaws (BRONJ) is an issue of particular concern. The majority of reported cases of bisphosphonate osteonecrosis have been subsequent to dental extractions, intraoral surgical intervention or mucosal trauma (15-18). BRONJ adversely affects quality of life, producing significant morbidity in afflicted patients (19), and at present, the action mechanism of bisphosphonates is unclear, and their effects on osteoclasts remain evasive.

The present study aimed to examine the direct effects of bisphosphonates on RANKL-stimulated osteoclast differentiation, RAW cells were cultured with bisphosphonates. To 



Figure 6. Pit assay. We investigated the resorption activity of osteoclasts treated with bisphosphonates (zoledronate or TRK-530; $10 \mu \mathrm{M}$ ) or distilled water (control group) using the Corning Osteo Assay Surface Plate. After 10 days of culture, plates were observed under a light microscope. (A) Control, (B) zoledronate treatment $(10 \mu \mathrm{M})$ and $(\mathrm{C}) \mathrm{TRK}-530$ treatment.

\section{A 3-day zoledronate}
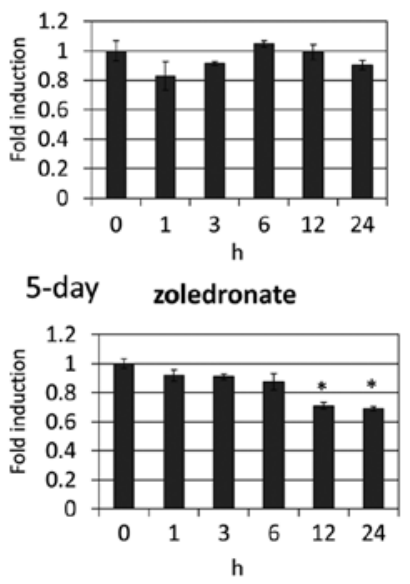

TRK-530



TRK-530

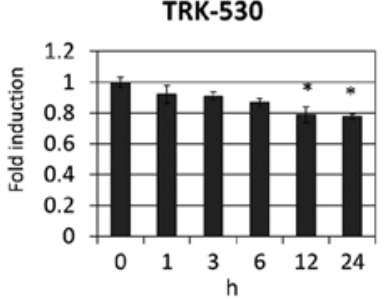

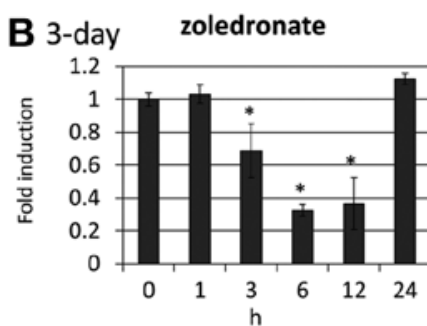

5-day zoledronate

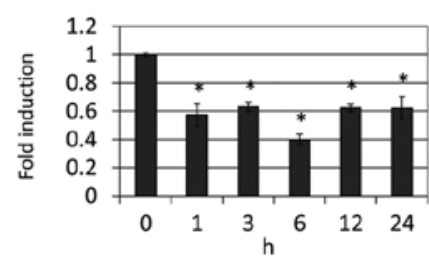

TRK-530

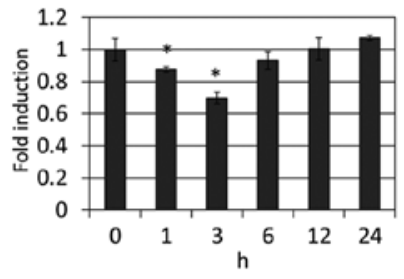

TRK-530

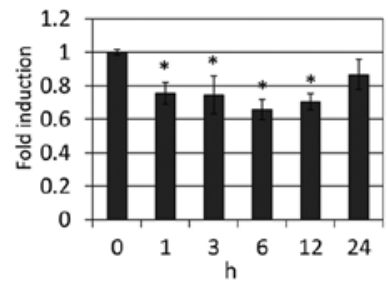

C 3-day zoledronate
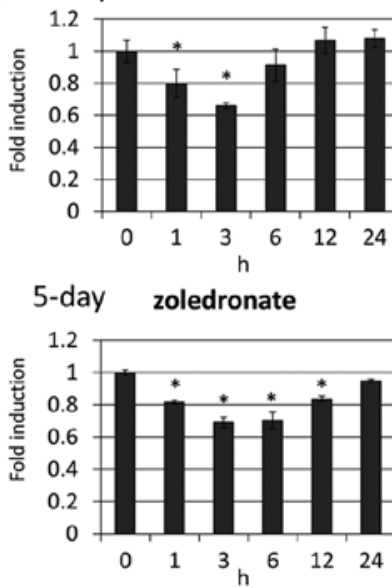

TRK-530

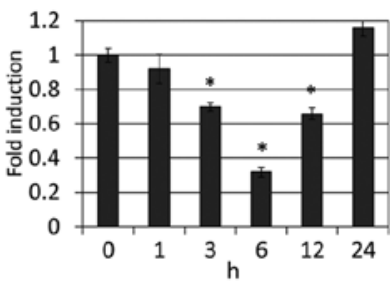

TRK-530

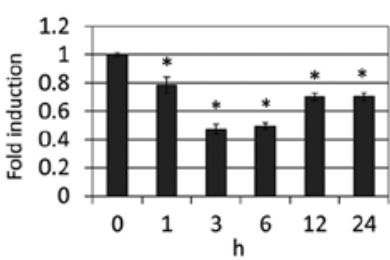

Figure 7. Effects of bisphosphonates on expression of osteoclastic differentiation genes and osteoclast-specific markers in RAW264.7 cells. After 3 or 5 days of culture, RAW264.7 cells were treated with bisphosphonates (zoledronate or TRK-530) $(10 \mu \mathrm{M})$, or were left untreated (control group), for $0,1,3,6,12$ or $24 \mathrm{~h}$. We analyzed the mRNA expression of target genes by real-time PCR analysis. Genes examined for mRNA expression were (A) NFATc1, (B) DC-STAMP and (C) TRAP. Results are shown as means \pm SD for four independent experiments. " $\mathrm{P}<0.05$, significant difference vs. controls.

investigate the effects of bisphosphonates on osteoclast differentiation, we treated cells with bisphosphonates after 3 days of culture, and to investigate the effects of bisphosphonates on mature osteoclasts, we treated cells after 5 days of culture. We found that bisphosphonates reduced the number of osteoclasts. Because the aim of this study was to investigate the effects of bisphosphonates on RANKL-induced osteoclastogenesis, we used RAW cells, which express endogenous RANK and can differentiate into osteoclasts following RANKL stimulation, in subsequent experiments. Addition of bisphosphonates to RAW cells undergoing osteoclast differentiation induced by RANKL led to a significant decrease in the number of osteoclasts in a bisphosphonate concentration-dependent (Fig. 3) and timedependent manner (Fig. 4). Similarly, the number of large 
A 3-day zoledronate


C 3-day zoledronate



5-day zoledronate



TRK-530

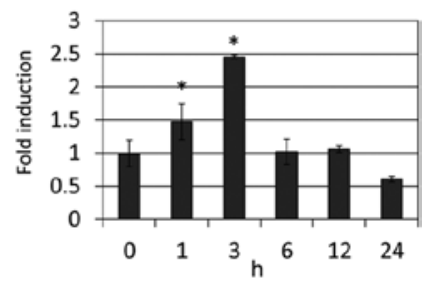

TRK-530

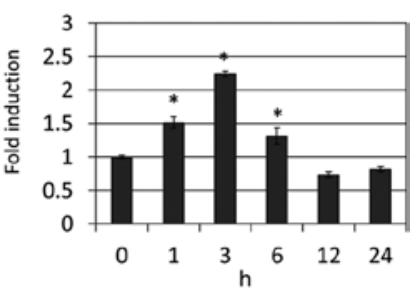

TRK-530



TRK-530

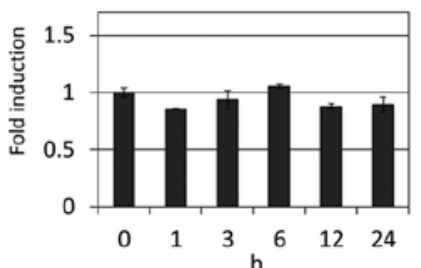

B 3-day Zoledronate
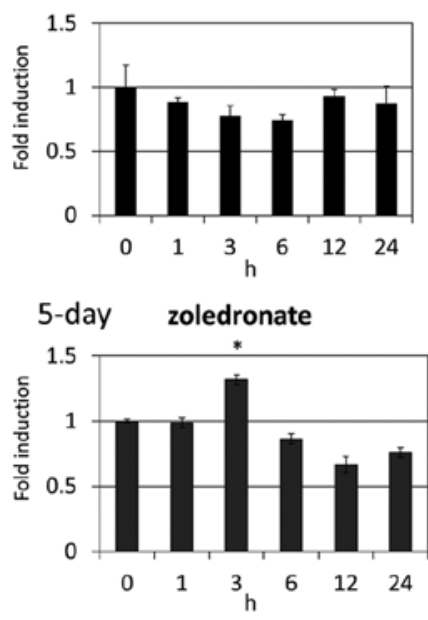

D 3-day zoledronate
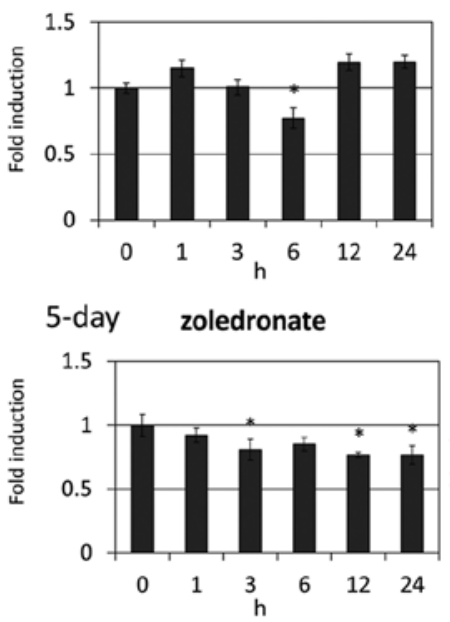



TRK-530

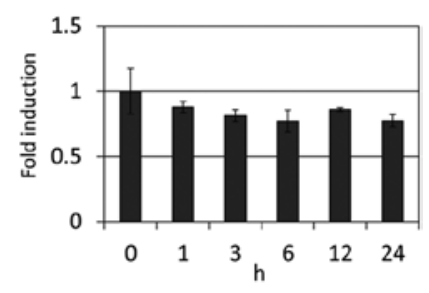

TRK-530

TRK-530

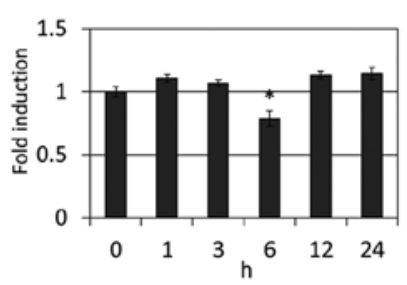

TRK-530

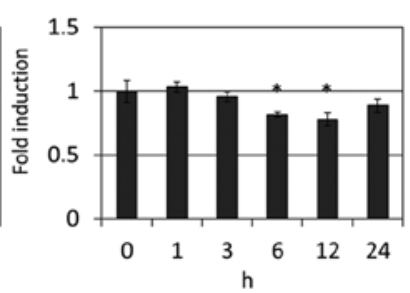

Figure 8. Effects of bisphosphonates on expression of genes related to apoptosis in RAW264.7 cells. After 3 or 5 days of culture, RAW264.7 cells were treated with bisphosphonates (zoledronate or TRK-530) $(10 \mu \mathrm{M}$ ), or were left untreated (control group), for 0, 1,3,6,12 or $24 \mathrm{~h}$. Genes examined for mRNA expression were (A) Fas, (B) caspase-3, (C) caspase-8 and (D) Bcl-xL. Results are shown as means \pm SD for four independent experiments. "P<0.05, significant difference vs. controls.

osteoclasts was suppressed by addition of bisphosphonates. These findings indicate that bisphosphonates inhibit osteoclast differentiation and fusion.

The cytotoxicity of bisphosphonates in RAW cells and osteoclasts was thus confirmed. Non-nitrogen-containing bisphosphonates were not cytotoxic and did not induce cell death in RAW cells, but were cytotoxic and induced cell death in osteoclasts (Fig. 5A). This indicates that the cytotoxicity of non-nitrogen-containing bisphosphonates is specific to osteoclasts. Meanwhile, although nitrogen-containing bisphosphonates were only slightly cytotoxic and induced cell death in RAW cells, they were severely cytotoxic and induced cell death in osteoclasts (Fig. 5B). This indicates that nitrogen-containing bisphosphonates were cytotoxic and induced cell death in both osteoclasts and RAW cells, although the effects were more marked in osteoclasts than in RAW cells. TRK-530, which does not contain nitrogen and was only recently developed, was not cytotoxic and did not induce cell death in RAW cells, but was cytotoxic and induced cell death in osteoclasts. Therefore, TRK-530-based therapy may have fewer adverse effects.
Generally, osteoclasts have resorption activity, and are thought to spend most of their lifetimes resorbing bone; thus, the preliminary stages and post-resorption stages must be relatively short when compared to the actual resorption stage (1). We investigated the resorption activity of osteoclasts treated with bisphosphonates (zoledronate and TRK-530). On pit assay, pit area was significantly lower after treatment with bisphosphonates as compared to controls (Fig. 6). These results suggest that bisphosphonates impair the resorption activity of osteoclasts.

We further investigated the effects of the bisphosphonates on the mRNA expression of osteoclast-associated genes in RAW cells. After 5 days of culture, expression of NFATc1 mRNA was decreased by bisphosphonate treatment (Fig. 7A). NFATc1 is a master regulator of RANKL-induced osteoclast differentiation and plays a pivotal role in osteoclast fusion and osteoclast activation via upregulation of various osteoclastrelated genes $(20,21)$. Therefore, suppression of NFATc1 mRNA by bisphosphonates indicates inhibition of osteoclast differentiation (20). NFATc1 has been shown to regulate the expression of osteoclast marker genes (1). After 5 and 3 days of 


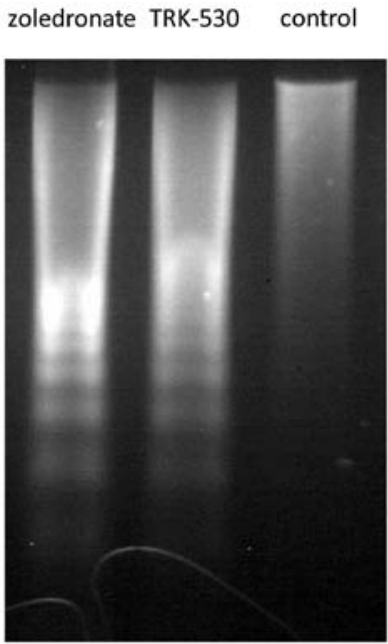

Figure 9. Detection of DNA fragmentation in agarose gels. After 3 days of culture, RAW264.7 cells were treated with bisphosphonates for $24 \mathrm{~h}$. DNA was visualized by ethidium bromide staining. Control was treated with distilled water.

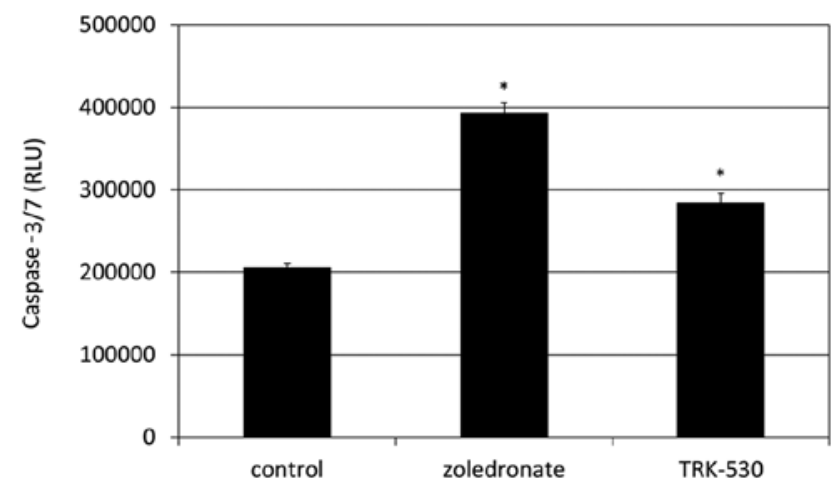

Figure 10. Caspase-3/7 activity. After 3 days of culture, bisphosphonates activated caspase-3/7. Luminescence was measured using a luminometer. Blank values were subtracted, and fold increase in activity was calculated based on activity measured from untreated cells. Control was treated with distilled water. Results are shown as means \pm SD for four independent experiments. ${ }^{*} \mathrm{P}<0.05$, significant difference vs. controls.

culture, bisphosphonates also inhibited the mRNA expression of TRAP, which is an osteoclast-specific marker (Fig. 7C), and this suppression corresponded to a bisphosphonate-induced decrease in the number of osteoclasts. Furthermore, recent studies have demonstrated that NFATc1 induced osteoclast fusion via upregulation of DC-STAMP (22), which is essential for cell-cell fusion in osteoclasts (23).

In this study, bisphosphonates reduced the expression of DC-STAMP mRNA after 5 and 3 days of culture (Fig. 7B). After 5 days of culture, bisphosphonates appeared to inhibit osteoclast differentiation and fusion via down-regulation of NFATc1 signaling. However, after 3 days of culture, as compared with the decrease in the number of osteoclasts, the mRNA expression of NFATc1 showed no significant differences when compared with controls. Although the mRNA expression of osteoclast marker genes did not always correlate with the number of osteoclasts $(24,25)$, this discrepancy suggested that other factors may be involved in the effects of bisphosphonates on osteoclast differentiation.
We further analyzed the mRNA expression of genes with reference to apoptosis. In general, bisphosphonates induce programmed cell death (apoptosis) (26). However, no previous studies have circumstantially assessed bisphosphonate-induced apoptosis in osteoclasts. After 3 and 5 days of culture, the genes responsible for apoptosis induction tended to increase, and the genes responsible for apoptosis inhibition tended to decrease (Fig. 8). We conducted further research on the apoptosis of osteoclasts by bisphosphonates using the detection of DNA fragmentation and caspase-3/7 activity. After 3 days of culture, treatment with bisphosphonates resulted in DNA fragmentation (Fig. 10) and significant increases in caspase-3/7 activity when compared to controls (Fig. 9). These findings clearly indicate that bisphosphonates induce apoptosis in osteoclasts.

In summary, we confirmed that bisphosphonates directly inhibit RANKL-stimulated osteoclast differentiation and fusion in RAW cells. It was also confirmed that bisphosphonates impair resorption activity and induce apoptosis in osteoclasts. Finally, the results confirmed that the effects of non-nitrogen-containing bisphosphonates are specific to osteoclasts, while nitrogen-containing bisphosphonates were cytotoxic and induced cell death in both osteoclasts and RAW cells.

\section{Acknowledgements}

We are grateful to Dr K. Shibata for technical advice and support. This study was supported in part by the Japanese Society for the Promotion of Science; Grants: Grant-in-Aid for Scientific Research (C) 22592274 to Y.Y., 21592584 to T.K. and 23592910 to K.T.

\section{References}

1. Väänänen HK and Laitala-Leinonen T: Osteoclast lineage and function. Arch Biochem Biophys 473: 132-138, 2008.

2. Boyle WJ, Simonet WS and Lacey DL: Osteoclast differentiation and activation. Nature 423: 337-342, 2003.

3. Yoshida H, Hayashi S, Kunisada T, et al: The murine mutation osteopetrosis is in the coding region of the macrophage colony stimulating factor gene. Nature 345: 442-444, 1990.

4. Lolli ML, Rolando B, Tosco P, et al: Synthesis and preliminary pharmacological characterisation of a new class of nitrogencontaining bisphosphonates (N-BPs). Bioorg Med Chem 18: 2428-2438, 2010.

5. Shinoda H, Takeyama S, Suzuki K, Murakami S and Yamada S: Pharmacological topics of bone metabolism: a novel bisphosphonate for the treatment of periodontitis. J Pharmacol Sci 106: 555-558, 2008.

6. Kimachi K, Kajiya H, Nakayama S, Ikebe T and Okabe K: Zoledronic acid inhibits RANK expression and migration of osteoclast precursors during osteoclastogenesis. Naunyn Schmiedebergs Arch Pharmacol 383: 297-308, 2011.

7. Kwak HB, Kim JY, Kim KJ, et al: Risedronate directly inhibits osteoclast differentiation and inflammatory bone loss. Biol Pharm Bull 32: 1193-1198, 2009.

8. Weinstein RS, Roberson PK and Manolagas SC: Giant osteoclast formation and long-term oral bisphosphonate therapy. N Engl J Med 360: 53-62, 2009.

9. Seo SW, Cho SK, Storer SK and Lee FY: Zoledronate reduces unwanted bone resorption in intercalary bone allografts. Int Orthop 34: 599-603, 2010.

10. Suzuki N, Yoshimura Y, Deyama Y, Suzuki K and Kitagawa Y: Mechanical stress directly suppresses osteoclast differentiation in RAW264.7 cells. Int J Mol Med 21: 291-296, 2008.

11. Ishida Y,Agata Y, Shibahara K and Honjo T: Induced expression of PD-1, a novel member of the immunoglobulin gene superfamily, upon programmed cell death. EMBO J 11: 3887-3895, 1992. 
12. Reginster JY, Malaise O, Neuprez A, Jouret VE and Close P Intermittent bisphosphonate therapy in postmenopausal osteoporosis: progress to date. Drugs Aging 24: 351-359, 2007.

13. Clarke NW: Skeletal preservation in prostate cancer: the changing role of the urologist. Eur Urol Suppl 5: 871-872, 2006.

14. Santini D, Vespasiani Gentilucci U, Vincenzi B, et al: The antineoplastic role of bisphosphonates: from basic research to clinical evidence. Ann Oncol 14: 1468-1476, 2003.

15. Ruggiero SL, Mehrotra B, Rosenberg TJ and Engroff SL: Osteonecrosis of the jaws associated with the use of bisphosphonates: a review of 63 cases. J Oral Maxillofac Surg 62: 527-534, 2004.

16. Marx RE,Sawatari Y,Fortin M and Broumand V: Bisphosphonateinduced exposed bone (osteonecrosis/osteopetrosis) of the jaws risk factors, recognition, prevention, and treatment. J Oral Maxillofac Surg 63: 1567-1575, 2005.

17. Dannemann C, Gratz KW, Riener MO and Zwahlen RA: Jaw osteonecrosis related to bisphosphonate therapy: a severe secondary disorder. Bone 40: 828-834, 2007.

18. Urade M, Tanaka N, Furusawa K, et al: Nationwide survey for bisphosphonate-related osteonecrosis of the jaws in Japan. J Oral Maxillofac Surg 69: 364-371, 2011.

19. Ferrara $S$ and Califano L: Current perspectives in the management of patients with drug-induced osteonecrosis of the maxilla: experience of the school of Naples Federico II. Age and Health Forum Catanzaro, Naples, pp5-7, 2009.
20. Takayanagi $\mathrm{H}$ : The role of NFAT in osteoclast formation (Review). NY Acad Sci 1116: 227-237, 2007.

21. Zhao Q, Wang X, Liu Y, He A and Jia R: NFATc1: functions in osteoclasts. Int J Biochem Cell Biol 42: 576-579, 2010

22. Kim K, Lee SH, Kim JH, Choi Y and Kim N: NFATc1 induces osteoclast fusion via upregulation of Atp6v0d2 and the dendritic cell-specific transmembrane protein (DC-STAMP). Mol Endocrinol 22: 176-185, 2008.

23. Yagi M, Miyamoto T, Sawatani Y, et al: DC-STAMP is essential for cell-cell fusion in osteoclasts and foreign body giant cells. J Exp Med 202: 345-351, 2005.

24. Kitami S, Tanaka H, Kawato T, et al: IL-17A suppresses the expression of bone resorption-related proteinases and osteoclast differentiation via IL-17RA or IL-17RC receptors in RAW264.7 cells. Biochimie 92: 398-404, 2010.

25. Shibata K, Yoshimura Y, Kikuiri T, et al: Effect of the release from mechanical stress on osteoclastogenesis in RAW264.7 cells. Int J Mol Med 28: 73-79, 2011

26. Hughes DE, Wright KR, Uy HL, et al: Bisphosphonates promote apoptosis in murine osteoclasts in vitro and in vivo. J Bone Miner Res 10: 1478-1487, 1995. 